\title{
Early Detection of Possible Outbreaks from Electronic Laboratory Reports
}

\author{
Hwa-Gan Chang*, Candace Noonan-Toly, Jian-Hua Chen and Bryon Backenson \\ New York State Department of Health, Albany, NY, USA
}

\section{Introduction}

New York State has implemented a statewide Electronic Clinical Laboratory Reporting System (ECLRS) to which laboratories can electronically submit test results for reportable conditions. The Communicable Disease Electronic Surveillance System (CDESS) was used by 57 Local Health Departments (LHDs) to transfer ECLRS information and initiate investigations. Currently over $98 \%$ of licensed clinical labs are reporting via ECLRS. Positive laboratory test results are required to confirm over $80 \%$ of communicable diseases and they are often the first indication of a disease. Early detection of disease outbreaks is important for timely implementation of disease prevention and control measures. The space-time permutation scan statistic only requires disease counts, event date and disease location, which are collected from ECLRS and can be used to detect potential disease outbreaks by identifying spatial-temporal lab report clusters.

\section{Methods}

Laboratory positive test results reported via ECLRS from 1/1/2011 through 7/31/2013 for seven communicable diseases: Campylobacter, Cryptospora, Giardia, Legionella, Pertussis, Salmonella, and Shigella were selected and de-duplicated by patient last name, first name, date of birth and specimen collection year. The space-time permutation scan statistic was applied for the period of 5/1/2013 through $7 / 31 / 2013$ using patient's zipcode as geographic level and ECLRS specimen collection date as the event date with baseline data from $1 / 1 / 2011$ to $4 / 30 / 2013$. The maximum cluster size was set as a circle with radius $<=20$ kilometer $(\mathrm{km})$ that could span up to 21 days. A table and map were generated if a possible cluster was detected with a p-value $<0.05$. LHDs transferred information from ECLRS to CDESS and entered the outbreak status of reported cases in CDESS when there was an outbreak or cluster cases. This data field was used to confirm the detection identified by scan statistic. The timeliness of detecting a cluster was calculated from specimen collection date to cluster identified date and was compared with the duration from specimen collection date to CDESS investigation start date .

\section{Results}

There were 2,050 patients with positive lab tests for 7 selected diseases reported via ECLRS during the study period. Table 1 indicates that 39 clusters were identified using space-time permutation scan statistic, $41 \%$ of them were confirmed clusters and the percentage varied by disease. The median duration from specimen collection date to cluster identified date ranged from 2 to 14 days and also varied by disease.

\section{Conclusions}

The space-time permutation scan statistic is easy to implement for detecting possible clusters from an electronic laboratory reporting system. The current study showed that this method can detect Camplobacter, Cryptospora, Pertussis, Salmonella and Shigellosis clusters earlier than traditional methods and has been set up as a routine procedure for identifying possible clusters. The space-time permutation scan statistic will be evaluated for other disease lab reports.
Table 1. Clusters Detected from Electronic Laboratory Reporting System, 5/1$7 / 31 / 2013, N Y$

\begin{tabular}{|c|c|c|c|c|}
\hline Disease & $\begin{array}{c}\text { No. of lab } \\
\text { tests }\end{array}$ & $\begin{array}{c}\text { No. of clusters } \\
\text { detected }\end{array}$ & $\begin{array}{c}\text { No. of confirmed } \\
\text { clusters }\end{array}$ & $\begin{array}{c}\text { Median days to identify } \\
\text { clusters }\end{array}$ \\
\hline Camplobacrter & 746 & 3 & 2 & 3 \\
\hline Cryptospora & 71 & 2 & 1 & 4 \\
\hline Giardia & 271 & 6 & 1 & 14 \\
\hline Legionella & 219 & 4 & 0 & 6 \\
\hline Pertussis & 244 & 15 & 7 & 7 \\
\hline Salmonella & 436 & 6 & 4 & 2 \\
\hline Shigella & 63 & 3 & 1 & \\
\hline
\end{tabular}

\section{Keywords}

ELR; Outbreak; Scan statistics

\section{*Hwa-Gan Chang}

E-mail: hgc04@health.ny.gov 